\title{
Hierarchical multimodal hub location problem with time-definite deliveries
}

\author{
Sibel A. Alumur ${ }^{\mathrm{a}, *}$, Hande Yaman ${ }^{\mathrm{b}}$, Bahar Y. Kara ${ }^{\mathrm{b}}$ \\ a Department of Industrial Engineering, TOBB University of Economics and Technology, Söğütözü 06560, Ankara, Turkey \\ ${ }^{\mathrm{b}}$ Department of Industrial Engineering, Bilkent University, Bilkent 06800, Ankara, Turkey
}

\section{A R T I C L E I N F O}

\section{Article history:}

Received 16 September 2011

Received in revised form 2 April 2012

Accepted 3 April 2012

\section{Keywords:}

Hub location

Time-definite delivery

Multimode transportation

Hierarchical network design

Valid inequalities

\begin{abstract}
A B S T R A C T
Hierarchical multimodal hub location problem is a cost-minimizing hub covering problem where two types of hubs and hub links, accounting for ground and air transportation, are to be established, while ensuring time-definite deliveries. We propose a mixed-integer programming formulation and perform a comprehensive sensitivity analysis on the Turkish network. We show that the locations of airport hubs are less sensitive to the cost parameters compared to the locations of ground hubs and it is possible to improve the service quality at not much additional cost in the resulting multimodal networks. Our methodology provides the means for a detailed trade-off analysis.
\end{abstract}

(c) 2012 Elsevier Ltd. All rights reserved.

\section{Introduction}

Hub facilities are present in various many-to-many distribution networks such as airline, cargo delivery, and telecommunications networks. In all of these networks, traffic between the demand nodes is routed via the hub facilities. Even though the basic structure of the traffic movement is the same, each application area has its additional requirements and specifications.

In this paper, we focus on the operational characteristics of cargo delivery networks. In a typical cargo delivery network, goods that need to be delivered are first collected at branch offices. The cargo processing operations, such as sorting, are carried out at hub facilities. These hub facilities are consolidation and dissemination centers. In hubs, cargo from different origins but similar destinations is collected together and re-routed according to their destinations. A package arriving at a hub can travel directly to its destination, if the destination branch office has a connection with this hub, or travels to the hub to which the destination is allocated. Due to managerial reasons, usually each branch office has connections with only one hub; that is, the in- and out-going traffic of each branch office is processed at a single hub.

Due to the competitive environment in the market, companies pay more attention to service levels. In cargo delivery, service level is primarily measured via delivery time, which is the time the parcel arrives at its destination. Cargo companies offer different delivery time promises, such as next day or second day delivery, to their customers.

'Next day delivery' or 'delivery within 24 h' is the current target for the cargo companies operating in Turkey. However, due to geographical distribution of the cities within Turkey and the structure of the highways, delivery within $24 \mathrm{~h}$ between all city pairs is not possible if only ground transportation is employed. Mainly due to the competitiveness in the sector, recently the cargo companies in Turkey investigate the costs and benefits of including airlines into their distribution networks. In particular,

\footnotetext{
* Corresponding author. Tel.: +90 312 2924136; fax: +90 3122924091.

E-mail addresses: salumur@etu.edu.tr (S.A. Alumur), hyaman@bilkent.edu.tr (H. Yaman), bkara@bilkent.edu.tr (B.Y. Kara).
} 
one of the cargo companies utilizes airline segments in its hub network and promises deliveries within $24 \mathrm{~h}$ between every origin-destination pair of Turkey. The company uses a star-shaped airline network that is rooted at the capital city Ankara.

Motivated by the operating characteristics of this company's service network, in this paper, we introduce a new hub location problem. In the sequel, we explain the operating structure of this type of service network in detail.

In this network, there are two different types of hubs: ground hubs and airport hubs. Each demand node is connected to a single hub via a highway segment. Additionally, each ground hub is connected to an airport hub via a highway segment, and each airport hub is connected to the central airport hub via an airline segment. Thus, the airline network has a star structure centered at the central airport hub. If two ground hubs are allocated to the same airport hub, then a direct highway segment can be established between these ground hubs. Even though the motivating example enforces a highway connection between any two ground hubs served by the same airport hub, we relax this assumption in our models and computational study, but, as extension we also discuss how to model this requirement.

Fig. 1 depicts a service network with eleven hub nodes (nodes $0, \ldots, 10$ ). In this figure, airport hubs are illustrated as triangles (nodes $1,2,3$ ), ground hubs as squares (nodes $4, \ldots, 10$ ), and the central airport hub is illustrated as a circle (node 0 ). Remaining unnumbered nodes are the demand nodes that are allocated to these hub nodes. Double lines represent airline connections, whereas single lines represent truck connections. Observe from Fig. 1 that the hub network has two levels: the first level is the star-shaped airline network and the second level is the union of mesh networks established for each airport hub.

In our hierarchical network, if two hubs are allocated to the same airport hub and if there is a direct highway segment in between, then the flow between these two hubs travels on this direct highway segment. If a direct highway segment is not established between two hubs that are allocated to the same airport hub, then the flow is routed using the highway segments allocating these hubs to their hub airport. On the other hand, if two hubs are allocated to two different airport hubs, then the traffic between them uses the highway segments connecting the hubs to their airport hubs and the airline segments connecting the associated airport hubs to the central airport hub. In the network depicted in Fig. 1, the flow between hub nodes 4 and 5 travels directly by using the highway segment in between, the flow from hub node 4 to hub node 6 follows the path $4 \rightarrow 1 \rightarrow 6$, whereas the traffic from hub node 4 to hub node 7 follows the path $4 \rightarrow 1 \rightarrow 0 \rightarrow 2 \rightarrow 7$. Note that, as pointed out by Smilowitz and Daganzo (2007), unlike airline passengers, cargo can be routed through more hubs if this results in economies of scale and cost savings.

In our application, both directions of a highway or airline segment incident to the central airport hub are served by the same vehicle. For instance, the airplane that travels from airport hub 1 to the central airport hub waits for the vehicles that arrive here from other nodes and then travels back to airport hub 1.

In this paper, we study the design of this type of a hierarchical multimodal hub network. We are given a set of demand nodes, a set of possible locations for ground hubs, a set of possible locations for airport hubs, the location of the central airport hub, the number of hubs to be opened, and the required cost and time parameters. The aim is to find the locations of the ground and airport hubs, the allocations of demand nodes to these hubs, the allocations of ground hubs to airport hubs and to route the flow to minimize the total transportation and operational costs while ensuring that each pair of demand nodes receive service within a predetermined time bound. We refer to this problem as the hierarchical multimodal hub location problem with time-definite deliveries (HMHL-TDD).

The hub location problem is first posed by O'Kelly $(1986,1987)$. Given a set of origin-destination pairs with positive flow, the hub location problem involves the decisions on the locations of the hubs and the allocations of the demand nodes to these hubs. O'Kelly (1987) proposes a cost minimizing formulation of the problem, which may be considered as the first model in the hub location literature.

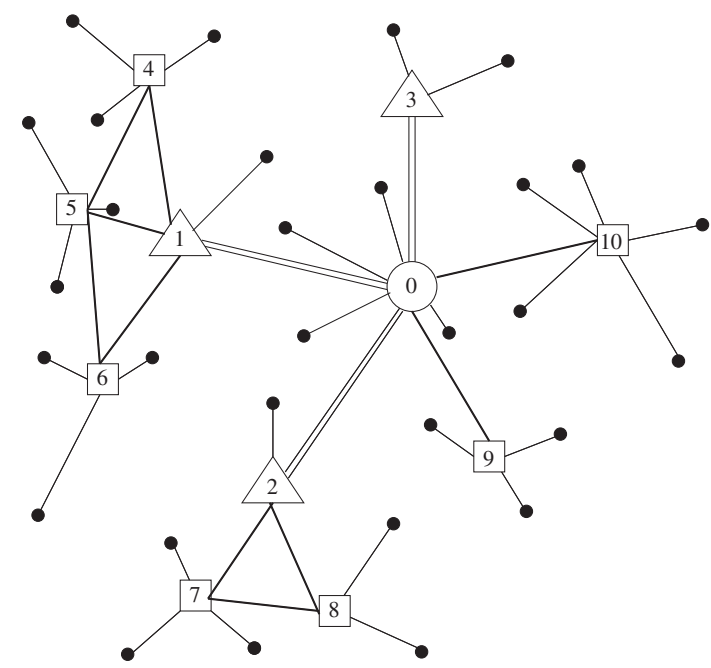

Fig. 1. A hierarchical multimodal hub network with eleven hub nodes. 
Campbell (1994) classifies hub location problems according to their optimization criteria: (i) minimization of the total transportation cost, $p$-hub median problem (which is the original model proposed in O'Kelly, 1987); (ii) minimization of the total transportation cost and the fixed cost of establishing hubs, uncapacitated/capacitated hub location problem, (iii) minimization of the maximum transportation cost, $p$-hub center problem, and (iv) minimization of the number of hubs while serving each pair within a predetermined bound, hub covering problem. The majority of the literature is devoted to the first two problems (see, e.g., Campbell, 1996; Ernst and Krishnamoorthy, 1996, 1998; O'Kelly et al., 1996; Sohn and Park, 1998; Boland et al., 2004; Hamacher et al., 2004; Labbé and Yaman, 2004; Labbé et al., 2005; Marín, 2005; Marín et al., 2006). For the $p$-hub center and hub covering problems, after Campbell (1994), the basics are developed by Kara and Tansel (2000, 2003), Ernst et al. (2005, 2009), Wagner (2008), and Meyer et al. (2009). For comprehensive surveys on hub location, the reader is referred to Campbell et al. (2002) and Alumur and Kara (2008).

In all the above mentioned studies, there is a basic understanding of the hub location problem. Given a set of nodes and positive flow between each origin-destination pair, determine the locations of hubs and the allocations of demand nodes to these hubs such that the flow between each origin-destination pair is routed through the hub nodes to optimize cost or a service related objective. It is customarily assumed that the hub network is complete; that is, once the flow arrives at a hub, it travels directly to the destination hub (if origin and destination are allocated to different hubs). This assumption leads to the fact that any package travels at most three segments, namely: origin-to-hub, hub-to-hub, and hub-to-destination. In the hub-to-hub portion of the journey, there is economies of scale, which is usually incorporated into the models by multiplying the corresponding cost coefficients with a scaling factor $\alpha$, where $0 \leqslant \alpha<1$.

Recently, there are some studies relaxing the complete hub network assumption and introducing hub network design decisions. Nickel et al. (2001), Yoon and Current (2008), Campbell et al. (2005a,b), Yaman et al. (2007), Labbé and Yaman (2008), Alumur and Kara (2009), Alumur et al. (2009, 2012), Calik et al. (2009), Campbell (2009), Contreras et al. (2009, 2010), and Yaman (2009) proposed hub location problems involving different hub network structures. Klincewicz (1998) and Gourdin et al. (2002) survey the studies on location problems with applications in telecommunications. We refer the reader to Şahin and Süral (2007) for a survey on hierarchical location problems.

The choice for mode of transportation within a hub network is initially discussed by O'Kelly and Lao (1991). In this study, allocation decisions using air and ground transportation are analyzed for two hubs at fixed locations. More recently, there are some studies considering hub location decisions in intermodal networks. Arnold et al. (2004) presented an integer programming model for locating rail/road terminals for freight transportation. Racunica and Wynter (2005) proposed a hub location model based on the uncapacitated hub location problem to increase the share of rail in intermodal transportation networks. Limbourg and Jourquin (2009) determined locations of rail-road container terminals using an iterative procedure based on both the $p$-hub median problem and the multimodal assignment problem. Ishfaq and Sox (2011) used uncapacitated hub location model with the inclusion of a service time constraint to determine the location of hubs within a rail-road intermodal network. Meng and Wang (2011) proposed a mathematical formulation to design an intermodal hub network for multi-type container transportation with multiple stakeholders. Although these studies capture the effect of using different transportation modes on hub locations, none of them considers different types of hub facilities to be located or the hierarchical hub network structure that we consider in this study.

Kara and Tansel (2001) focus on the cargo delivery sector applications of the hub location problem. The authors observe that 'synchronization' is crucial in determining the departure times. The departing vehicles from hubs should wait for the incoming vehicles. The authors pose the latest arrival hub location problem as the hub location problem in which departure time synchronization is also incorporated. Later, Tan and Kara (2007) studied the latest arrival hub covering problem and Yaman et al. (2007) incorporated stopovers in the latest arrival hub location problem. In all of the studies on latest arrival type hub location problems, the hub network is assumed to be complete and only a single transportation mode is considered.

In the studies with "time-definite deliveries", the service time between each origin-destination pair needs to be within a service level guarantee. Lin et al. (2004) determine the fleet sizes and schedules while ensuring a given service level. Smilowitz and Daganzo (2007) study the design of hierarchical integrated package distribution networks with multiple service levels using air and ground transportation. They minimize transportation costs, composed of fixed vehicle costs and variable operating costs, and facility costs, composed of fixed terminal charges, handling costs and storage expenses, using continuum approximation techniques. Chen et al. (2008) study a tree structured network where they seek to minimize the sum of the violations of the delivery guarantees. Campbell (2009) minimizes the total transportation cost while ensuring the time-definite transportation by considering feasible assignments only. Sim et al. (2009) consider stochastic travel times. Other examples are Yaman (2009) where a hierarchical hub network is designed and Alumur et al. (2009) and Alumur and Kara (2009) where incomplete hub networks are allowed. More recently, Lin (2010) studies a hub network design problem where each node is connected through a secondary route to its designated hub, Yaman (2011) studies the effect of different allocation strategies and Yaman et al. (2012) consider a cost minimization problem for the cargo sector in which the release times are to be determined in addition to the hub locations.

The hierarchical multimodal hub location problem HMHL-TDD introduced in this paper is a cost minimization hub location problem with time-definite deliveries over a hub network that contains airline and highway segments.

In this paper, we introduce a new hub location problem, propose a mathematical formulation of the problem, and present computational analysis to evaluate the solution potential of the proposed model. Results obtained for the Turkish network data are presented with emphasis on the effects of the choice of parameters and the delivery time restrictions on total cost and the locations of the hub nodes. 
The organization of the paper is as follows: In the next section, we propose a mixed integer programming formulation of the problem. In Section 3, we present the results of our computational study on the Turkish network. The paper ends with some concluding remarks presented in Section 4.

\section{Problem formulation}

In this section, we introduce a mathematical formulation for HMHL-TDD. The parameters of the problem are as follows:

Let $D$ denote the set of demand points, $H \subseteq D$ the set of possible locations for hubs, $A \subseteq H$ the set of possible locations for airport hubs, and $0 \in A$ denote the central airport hub.

We denote the number of hubs that are going to be established (including the central airport hub) by $p$. The traffic demand from node $i \in D$ to node $j \in D$ is denoted by $w_{i j}$. We let $t_{i j}$ and $t_{i j}^{P}$ denote the time to travel from node $i$ to node $j$ by truck and by airplane, respectively. If both nodes are hubs, then the travel time for a truck is reduced by a factor of $\alpha$, where $0 \leqslant \alpha<1$. If node $j \in A \backslash\{0\}$ is an airport hub then there is a loading/unloading time of $m_{j}$ units. The loading and unloading time at the central airport hub is $\hat{m}_{0}$ units. Finally, all cargo should reach its destination by time $T$. We assume that all travel times and loading/unloading times are nonnegative.

We group and explain the variables and the constraints of our mathematical formulation below:

$H u b$ locations and allocations: We define $x_{i j}$ to be 1 if demand point $i \in D$ is allocated to hub $j \in H$ and to be 0 otherwise. With this definition, $x_{j j}$ is 1 when a hub is established at node $j \in H$. We additionally define $y_{j l}$ to be 1 if hub $j \in H$ is allocated to airport hub $l \in A$ and 0 otherwise. Similarly, $y_{j j}$ is 1 if an airport hub is established at $j \in A$.

The following constraints model the allocation and location requirements:

$$
\begin{array}{lc}
\sum_{j \in H} x_{i j}=1 & \forall i \in D \\
x_{i j} \leqslant x_{j j} & \forall i \in D, j \in H \\
\sum_{l \in A} y_{j l}=x_{j j} & \forall j \in H \\
y_{j l} \leqslant y_{l l} & \forall j \in H, l \in A \\
\sum_{j \in H} x_{j j}=p & \\
y_{00}=1 & \\
x_{i j} \in\{0,1\} & \forall i \in D, j \in H \\
y_{j l} \in\{0,1\} & \forall j \in H, l \in A .
\end{array}
$$

Constraints (1) ensure that each demand node is allocated to a single hub. By Constraints (2), if a demand node is allocated to a certain node, then that node should be a hub node. Due to Constraints (3), a hub is allocated to exactly one airport hub. If a hub is allocated to an airport hub, then this airport hub must be established by Constraints (4). We set the total number of hubs to be established to $p$ by Constraint (5). Constraint (6) ensures that the central airport hub is opened. Finally, Constraints (7) and (8) define the binary variables.

Design of the hub network: In the hub network to be designed, the airline connections form a star network. That is, each airport hub is to be connected to the central airport by a direct flight and no direct connection between non-central airport hubs is allowed. We do not need to have additional decision variables or constraints for the design of this airline network since the information on a node becoming an airport hub or not is sufficient for this star structured design.

All the connections in the network, other than the ones within the star shaped airline network, utilize trucks. There must be a direct truck connection between a hub and its airport hub. Other truck connections in the hub network can only exist between hub nodes that are allocated to the same airport hub. The following constraints model these hub network design restrictions.

First, we define $z_{j k}^{l}$ to be 1 if there is a truck connection from hub $j \in H$ to hub $k \in H \backslash\{j\}$ and if $j$ and $k$ are both allocated to the same airport hub $l \in A$, and to be 0 otherwise. We include the constraints:

$$
\begin{array}{ll}
z_{j l}^{l}=y_{j l} & \forall j \in H, l \in A \backslash\{j\} \\
z_{l j}^{l}=y_{j l} & \forall j \in H, l \in A \backslash\{j\} \\
z_{j k}^{l} \leqslant y_{j l} & \forall j \in H, k \in H \backslash\{j\}, l \in A \\
z_{j k}^{l} \leqslant y_{k l} & \forall j \in H, k \in H \backslash\{j\}, l \in A \\
z_{j k}^{l} \in\{0,1\} & \forall j \in H, k \in H \backslash\{j\}, l \in A .
\end{array}
$$

Transfers between hubs and their designated airport hubs require the use of trucks; this is expressed in Constraints (9) and (10). A direct truck connection can exist between two hubs only if these two hubs, say $j$ and $k$, are allocated to the same airport hub, say $l$; i.e., if both $y_{j l}$ and $y_{k l}$ are equal to 1 . This is modeled by Constraints (11) and (12). 
If it is required that there must be a direct truck connection between all hubs that are allocated to the same airport hub, to form a complete sub-network (clique) for each airport hub, the following constraints should be included in the model:

$$
z_{j k}^{l} \geqslant y_{j l}+y_{k l}-y_{l l} \quad \forall j \in H, k \in H \backslash\{j\}, l \in A .
$$

Routing the flow: In order to route the flow on the hub network to be designed, we need to define two additional sets of decision variables. We use a multicommodity flow formulation as the one of Ernst and Krishnamoorthy (1996) for the $p$-hub median problem. The variable $f_{j k}^{i}$ is the flow that originates at node $i \in D$ and that travels from hub $j \in H$ to hub $\left.k \in H \backslash j\right\}$ using trucks. We define $g_{j 0}^{i}$ and $g_{0 j}^{i}$ to be the flow that originates at node $i$ and that travels on the airplane from airport hub $j \in A \backslash\{0\}$ to the central airport hub and from the central airport hub to airport hub $j$, respectively.

The following sets of constraints ensure that the traffic demand is routed using the available links.

$$
\begin{aligned}
& \sum_{k \in H \backslash\{j\}} f_{j k}^{i}-\sum_{k \in H \backslash\{j\}} f_{k j}^{i}=\sum_{s \in D} w_{i s}\left(x_{i j}-x_{s j}\right) \quad \forall i \in D, j \in H \backslash A \\
& \sum_{k \in H \backslash\{j\}} f_{j k}^{i}+g_{j 0}^{i}-g_{0 j}^{i}-\sum_{k \in H \backslash j\}} f_{k j}^{i}=\sum_{s \in D} w_{i s}\left(x_{i j}-x_{s j}\right) \quad \forall i \in D, j \in A \backslash\{0\} \\
& \sum_{k \in H \backslash\{0\}} f_{0 k}^{i}+\sum_{j \in A \backslash\{0\}} g_{0 j}^{i}-\sum_{j \in A \backslash\{0\}} g_{j 0}^{i}-\sum_{k \in H \backslash\{0\}} f_{k 0}^{i}=\sum_{s \in D} w_{i s}\left(x_{i 0}-x_{s 0}\right) \quad \forall i \in D \\
& f_{j k}^{i} \leqslant \sum_{s \in D} \sum_{l \in A} w_{i s} z_{j k}^{l} \quad \forall i \in D, j \in H, k \in H \backslash\{j\} \\
& g_{j 0}^{i}+g_{0 j}^{i} \leqslant \sum_{s \in D} w_{i s} y_{j j} \quad \forall i \in D, j \in A \backslash\{0\} \\
& f_{j k}^{i} \geqslant 0 \quad \forall i \in D, j \in H, k \in H \backslash\{j\} \\
& g_{j 0}^{i}, g_{0 j}^{i} \geqslant 0 \quad \forall i \in D, j \in A \backslash\{0\} .
\end{aligned}
$$

Constraints (15) are the flow balance constraints at ground hubs. Similarly, Constraints (16) are the flow balance constraints at the airport hubs considering the flow using both trucks and airplanes. Constraints (17) are the flow balance constraints at the central airport hub.

Constraints (18) ensure that the flow using trucks can be routed only on the established truck connections. Similarly, by Constraints (19) the flow using airplanes can be routed only on the established airplane connections. Constraints (20) and (21) are the non-negativity constraints for the flow variables.

Time restrictions: Finally, we model the requirement that all cargo should reach its destination within $T$ units of time. To this end, we define the following decision variables.

For a hub $j, r_{j}$ denotes the earliest time that a truck or an airplane can depart from hub $j$ towards other hubs. The earliest time that all the trucks from the demand nodes and ground hubs, and the airplanes from the airport hubs arrive at the central airport hub is denoted by $r_{0}$.

For each hub $j, \bar{r}_{j}$ denotes the longest time needed to deliver the cargo to the demand nodes allocated to hub $j$. On the other hand, $\bar{r}_{0}$ denotes the longest time needed to deliver the cargo from the central airport hub to all hubs and demand nodes.

We set $m_{0}=0$.

The following constraints model the time restrictions:

$$
\begin{aligned}
& r_{j} \geqslant t_{i j} x_{i j} \quad \forall i \in D, j \in H \\
& r_{0} \geqslant r_{j}+\sum_{l \in A}\left(\alpha t_{j l}+m_{l}+t_{l 0}^{p}\right) y_{j l} \quad \forall j \in H \\
& \bar{r}_{j} \geqslant t_{j i} x_{i j} \quad \forall i \in D, j \in H \\
& \bar{r}_{0} \geqslant \sum_{l \in A}\left(t_{0 l}^{P}+m_{l}+\alpha t_{l j}\right) y_{j l}+\bar{r}_{j} \quad \forall j \in H \\
& r_{j}+\sum_{l \in A} \alpha t_{j k} z_{j k}^{l}+\bar{r}_{k} \leqslant T \quad \forall j \in H, k \in H \backslash\{j\} \\
& r_{0}+\hat{m}_{0}+\bar{r}_{0} \leqslant T .
\end{aligned}
$$

The main idea behind Constraints (22)-(27) is that a truck or an airplane departing from a hub needs to wait for all the incoming flow. These time restriction constraints can be explained via Fig. 1. The trucks or airplanes leaving each of the hub nodes $\{0, \ldots, 10\}$ must wait for the flow coming from the demand nodes allocated to them. For example, the truck leaving hub node 4 towards hub node 5 or hub node 1 must wait for the flow arriving from the three demand nodes allocated to it. Thus, the earliest time that a truck can leave hub node 4 is determined by Constraints (22). 
The ready time of all the cargo at the central airport hub is determined by Constraints (22) and (23). Constraints (22) ensure that the central airport hub waits for the flow arriving from the demand nodes allocated to it. By Constraints (23), the central airport hub waits for the trucks and airplanes arriving from all the hub nodes. In our example, the central airport hub waits for the four demand nodes allocated to it by Constraints (22), for the trucks arriving from hub nodes 9 and 10 , and also for the airplanes arriving from airport hubs 1, 2, and 3, by Constraints (23).

Constraints (24) calculate the longest time needed to deliver the cargo from each hub to the demand nodes allocated to it. The longest time needed to deliver the cargo from the central airport hub to all hubs and demand nodes is calculated via Constraints (25).

Constraints (26) ensure that the cargo transported using the direct truck connections between the hub nodes must be delivered within the time limit. We remark here that Constraints (26) are redundant if the travel times satisfy the triangle inequality.

Finally, Constraints (27) ensure that the longest time to deliver the cargo between any origin-destination pair is within the time limit $T$.

In case the travel times are symmetric, there exists an optimal solution with $r_{j}=\bar{r}_{j}$ for all $j \in H$. Hence, the variables $\bar{r}_{j}$ 's and Constraints (24) and (25) can be dropped from the model and Constraints (26) and (27) can be replaced with

$$
\begin{aligned}
& r_{j}+\sum_{l \in A} \alpha t_{j k} z_{j k}^{l}+r_{k} \leqslant T \quad \forall j \in H, k \in H \backslash\{j\} \\
& 2 r_{0}+\hat{m}_{0} \leqslant T .
\end{aligned}
$$

If the demand points are over a large geographical area, there may be time zone differences between them. For such cases, the Constraints (22)-(27) should be revised to include the time zone differences. The revision is simply to add (or subtract) the time gained (or lost) due to time zone differences to the arrival time (Kara and Tansel, 2001).

Objective function: The objective function of our problem is to minimize the total transportation and operational costs. We define the following cost parameters:

$c_{i j}$ : $\quad$ the unit routing cost from $i \in D$ to $j \in D$ if one of the nodes is a non-hub node and the other node is its hub

$c_{j k}^{T}$ : $\quad$ the unit routing cost from $j \in H$ to $k \in H$ on a truck if both nodes are hubs

$c_{j 0}^{P}$ : $\quad$ the unit routing cost from node $j \in A \backslash\{0\}$ to node 0 on an airplane

$c_{0 j}^{P}$ : $\quad$ the unit routing cost from node 0 to node $j \in A \backslash\{0\}$ on an airplane

$C_{i j}$ : $\quad$ the fixed cost of operating an allocation connection between node $i \in D$ and hub $j \in H$

$C_{j k}^{T}$ : $\quad$ the fixed cost of operating a truck connection from hub $j \in H$ to hub $\left.k \in H \backslash j\right\}$

$C_{j}^{P}$ : $\quad$ the fixed cost of operating an airline connection between airport hub $j \in A \backslash\{0\}$ and the central airport hub

Using the above parameters, the objective function is expressed as:

$$
\begin{aligned}
& \operatorname{Min} \sum_{i \in D}\left(\sum_{j \in H}\left(c_{i j} \sum_{s \in D} w_{i s}+c_{j i} \sum_{s \in D} w_{s i}\right) x_{i j}+\sum_{j \in H} \sum_{k \in H \backslash\{j\}} c_{j k}^{T} f_{j k}^{i}+\sum_{j \in A \backslash\{0\}}\left(c_{j 0}^{P} g_{j 0}^{i}+c_{0 j}^{P} g_{0 j}^{i}\right)\right)+\sum_{i \in D} \sum_{j \in H} C_{i j} x_{i j}+\sum_{l \in A} \sum_{j \in H} \sum_{k \in H \backslash\{j\}} C_{j k}^{T} z_{j k}^{l} \\
& +\sum_{j \in A \backslash\{0\}} C_{j}^{P} y_{j j} .
\end{aligned}
$$

The first two terms in the objective function (30) sum the transportation costs on the allocation segments, the third term calculates the transportation costs between hub nodes using truck connections, the fourth and fifth terms, on the other hand, calculate the transportation costs using airplanes. The last three terms calculate the fixed costs of operating truck and airplane connections. The costs and the demand are in commensurate units of time so that the overall objective is the minimization of total cost per some unit of time.

A mixed-integer programming formulation of HMHL-TDD consists of the objective function (30) and the Constraints (1)(13) and (15)-(27). In the worst case, $|D|=|H|=|A|=n$ and the model has $O\left(n^{3}\right)$ binary variables, $O\left(n^{3}\right)$ continuous variables, and $O\left(n^{3}\right)$ constraints.

Before concluding this section, we present some variable fixing rules and valid inequalities based on travel times and the delivery time bound.

For $j \in H$ and $l \in A$, if $\alpha t_{j l}+m_{l}+t_{l 0}^{P}+\hat{m}_{0}+t_{0 l}^{P}+m_{l}+\alpha t_{l j}>T$, then if node $j$ becomes a hub, it cannot be allocated to the airport hub $l$. Hence, we can set $y_{j l}=0$ and $z_{j k}^{l}=z_{k j}^{l}=0$ for all $k \in H \backslash\{j\}$.

For $j \in H, k \in H$ such that $j<k$, and $l \in A$, suppose that nodes $j$ and $k$ are hubs and they are both allocated to the airport hub l. If $\alpha t_{j l}+m_{l}+t_{l 0}^{P}+\hat{m}_{0}+t_{0 l}^{P}+m_{l}+\alpha t_{l k}>T$ (the travel time from $j$ to $k$ exceeds $T$ ) or $\alpha t_{k l}+m_{l}+t_{l 0}^{P}+\hat{m}_{0}+t_{0 l}^{P}+m_{l}+\alpha t_{l j}>T$ (the travel time from $k$ to $j$ exceeds $T$ ), then such an allocation violates the delivery time constraints. As a result, the inequality

$$
y_{j l}+y_{k l} \leqslant y_{l l}
$$

is valid. Moreover, as $y_{j l}$ and $y_{k l}$ cannot take value 1 at the same time, we can set $z_{j k}^{l}=z_{k j}^{l}=0$. Inequality (31) dominates Constraint (4). 
For $j \in H, k \in H \backslash\{j\}$, and $l \in A$, we define $A_{j k l}=\left\{q \in A: \alpha t_{j l}+m_{l}+t_{l 0}^{P}+\hat{m}_{0}+t_{0 q}^{P}+m_{q}+\alpha t_{q k}>T\right.$ or $\alpha t_{k q}+m_{q}+t_{q 0}^{P}+\hat{m}_{0}+$ $\left.t_{0 l}^{P}+m_{l}+\alpha t_{l j}>T\right\}$. If hub $j$ is allocated to airport hub $l$, then $k$ cannot be allocated to any airport hub in the set $A_{j k l}$ since otherwise the travel time from $j$ to $k$ or from $k$ to $j$ exceeds the bound $T$. Hence the inequality

$$
y_{j l}+\sum_{q \in A_{j k l}} y_{k q} \leqslant 1
$$

is a valid inequality.

We also define $A_{j k l}^{->}=\left\{q \in A: \alpha t_{j l}+m_{l}+t_{l 0}^{P}+\hat{m}_{0}+t_{0 q}^{P}+m_{q}+\alpha t_{q k}>T\right\} \quad$ and $\quad A_{j k l}^{<-}=\left\{q \in A: \alpha t_{k q}+m_{q}+t_{q 0}^{P}+\hat{m}_{0}+\right.$ $\left.t_{0 l}^{P}+m_{l}+\alpha t_{l j}>T\right\}$. Note that $A_{j k l}^{->} \cup A_{j k l}^{<-}=A_{j k l}$. Let $\bar{A}_{j l}^{->}=\left\{q \in A: \alpha t_{j q}+m_{q}+t_{q 0}^{P} \geqslant \alpha t_{j l}+m_{l}+t_{l 0}^{P}\right\}$ and $\bar{A}_{j l}^{<-}=\left\{t_{0 q}^{P}+m_{q}+\right.$ $\left.\alpha t_{q j} \geqslant t_{0 l}^{P}+m_{l}+\alpha t_{l j}\right\}$. The set $\bar{A}_{j l}^{->}$is the set of airport hubs to which the travel time from hub $j$ is not less than its travel time to airport hub $l$. Hence if hub $j$ is allocated to any airport hub in set $\bar{A}_{j l}^{->}$, then $k$ cannot be allocated to any airport hub in the set $A_{j k l}^{->}$since otherwise the travel time from $j$ to $k$ exceeds the bound $T$. A similar argument can be made for sets $\bar{A}_{j l}^{<-}$and $A_{j k l}^{<-}$base on the travel time from $k$ to $j$. Then the inequalities

$$
\sum_{q \in \bar{A}_{j l}^{->}} y_{j q}+\sum_{q \in A_{j k l}^{->}} y_{k q} \leqslant 1
$$

and

$$
\sum_{q \in \bar{A}_{j l}^{<-}} y_{j q}+\sum_{q \in A_{j k l}^{<-}} y_{k q} \leqslant 1
$$

are valid inequalities.

Note that if the travel times are symmetric, then $A_{j k l}^{->}=A_{j k l}^{<-}=A_{j k l}$ and $\bar{A}_{j l}^{->}=\bar{A}_{j l}^{<-}=\bar{A}_{j l}$. In this case, the inequality

$$
\sum_{q \in \bar{A}_{j l}} y_{j q}+\sum_{q \in A_{j k l}} y_{k q} \leqslant 1
$$

is valid and dominates inequality (32).

Finally, let $i \in D$ and $j \in H$ and define $A_{i j}=\left\{l \in A: t_{i j}+\alpha t_{j l}+m_{l}+t_{l 0}^{P}+\hat{m}_{0}+t_{0 l}^{P}+m_{l}+\alpha t_{l j}+t_{j i}>T\right\}$. If node $i$ is allocated to hub node $j$, then $j$ cannot be allocated to any hub airport in the set $A_{i j}$. Hence, the inequality

$$
x_{i j}+\sum_{l \in A_{i j}} y_{j l} \leqslant x_{j j}
$$

is a valid inequality. Note that this inequality dominates Constraint (2).

\section{Application in Turkey}

We applied our hierarchical multimodal hub location model with time-definite deliveries on the Turkish network. The data on the distances between 81 cities of Turkey, the travel times using ground transportation, and the traffic demands are presented in OR Library, Beasley (1990). We note here that the data on the distances is updated due to construction of new highways within the country and we are using the most recent data presented in the link given in the OR Library.

The data on the candidate locations of the hub nodes is gathered from the cargo company. The company currently operates 22 hubs in order to serve 81 cities of Turkey within $24 \mathrm{~h}$. In constructing our test bed, we have chosen the company's 22 hub locations to be the potential hub set $H$. Fig. 2 depicts these 81 cities and 22 potential hub locations on the map of Turkey (the numbers represent the license plate codes by which the corresponding cities are commonly referred to in Turkey).

There is an airport in all the cities in the potential hub set, except three $(3,68$, and 81$)$. So, we took these 19 cities as the potential airport hub set $A$. The central airport hub is located in Ankara (6) where the cargo company has its major sorting center (Fig. 2).

The time discount factor $\alpha$ is taken as 0.9 (Tan and Kara, 2007). The travel times by airplane between the central airport Ankara and each airport in the set $A$ is estimated by assuming that the airplanes travel at a speed of $700 \mathrm{~km} / \mathrm{h}$. The loading/ unloading time at an airport hub excluding the central airport hub, $m_{j}$, is taken as $30 \mathrm{~min}$, whereas the loading and unloading time at the central airport hub, $\hat{m}_{0}$, is taken as $120 \mathrm{~min}$.

There are different routing and fixed cost parameters in the objective function of the model. We estimated realistic values for these parameters based on the interviews with the company representatives. In the current operating structure of the cargo company, a small truck operates between a demand node and a hub, whereas a trailer operates between two hubs. The unit routing costs per distance between hub nodes on truck connections are about 0.75 times the unit routing costs between a non-hub node and a hub node. On the other hand, the fixed cost of operating a small truck per unit distance is around $80 \%$ of the fixed cost of operating a trailer. The company representatives estimated the costs for routing unit flow on airline connections to be negligible compared to the fixed cost of operating an airplane. The cost of operating an airline 


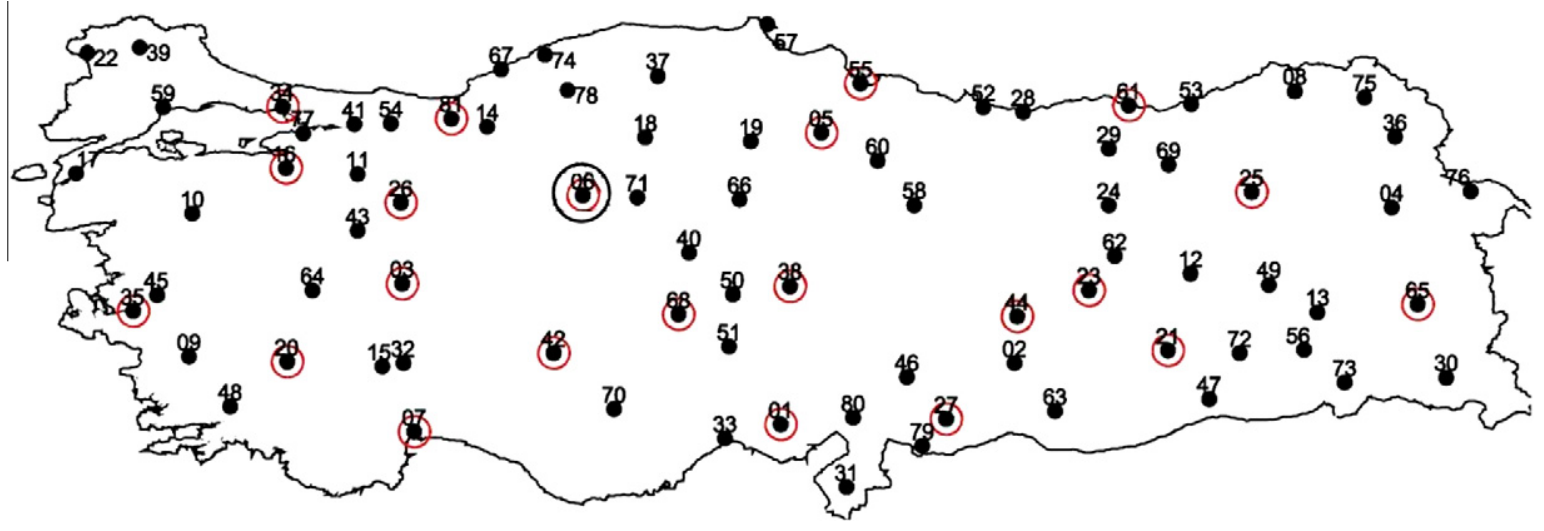

Fig. 2. 81 Cities, 22 potential hub locations, and the central airport hub on the Turkish network.

connection between an airport hub and Ankara is estimated as 3500. We varied the values of the fixed costs of operating truck and airline connections in order to observe the effects on the optimal solutions.

We took our runs on a server with $2.6 \mathrm{GHz}$ AMD Opteron 252 processor and $2 \mathrm{~GB}$ of RAM operating under the system CentOS and we used the optimization software CPLEX version 11.2.

Initially, we tested the performance of the variable fixing rules and valid inequalities introduced at the end of the previous section. For this analysis, we solved the model by CPLEX with and without the inclusion of valid inequalities and compared the results. While solving the model with the valid inequalities, we included all sets of inequalities developed for the symmetric data since the data on the Turkish network is symmetric. We put a time limit of two hours and compared the gap of the linear programming relaxations, CPU time requirements by CPLEX, and the numbers of nodes in the branch-and-bound tree. The results are summarized in Table 1.

The first two columns in Table 1 report the instance parameters: the time limit and the total number of hubs to be established. The columns indicated by 'No valid inequalities' correspond to the solutions without the inclusion of any valid inequalities, whereas the columns indicated by 'All valid inequalities' list the resulting solutions with the inclusion of all valid inequalities. The columns indicated by 'LP gap' report the gap of the linear programming relaxation from the optimal value and the rows indicated by 'CPU time' report the CPU time requirement in seconds by CPLEX. For each instance with and without the inclusion of valid inequalities, 'Nodes' columns list the number of nodes in the branch-and-bound tree reported by CPLEX. The last column in Table 1 calculates the percentage of improvement in the CPU times when using the valid inequalities.

When we compare the LP relaxations, observe from Table 1 that LP gaps are smaller with the valid inequalities in all the instances. However, the difference between the values of the two LP gaps are not very significant in some instances. For example, when $T=24, p=2$ and $T=23, p=4$. In general, the difference in the LP relaxation values increase with tighter time bounds. As expected, this shows that the valid inequalities are more effective with tighter service levels.

All the instances in Table 1 are solved to optimality within two hours. All the instances, except the second and third, are solved more quickly with the inclusion of the valid inequalities. At the instance with $T=24$ and $p=3$, the model with the valid inequalities required around $45 \mathrm{~s}$ more to be solved to optimality. Similarly, the instance with $T=23$ and $p=3$ required around 20 more seconds with the valid inequalities. On the other hand, as it can be observed from the last column of Table 1 ,

Table 1

Performance of the valid inequalities.

\begin{tabular}{|c|c|c|c|c|c|c|c|c|}
\hline \multirow[t]{2}{*}{$T(\mathrm{~h})$} & \multirow[t]{2}{*}{$p$} & \multicolumn{3}{|c|}{ No valid inequalities } & \multicolumn{3}{|c|}{ All valid inequalities } & \multirow[t]{2}{*}{ CPU time improvement (\%) } \\
\hline & & LP gap (\%) & CPU times (s) & Nodes & LP gap (\%) & $\mathrm{CPU}$ times $(\mathrm{s})$ & Nodes & \\
\hline 24 & 2 & 1.89 & 11.82 & 0 & 1.52 & 7.37 & 0 & 37.65 \\
\hline 24 & 3 & 5.33 & 57.20 & 29 & 4.66 & 101.33 & 17 & -43.55 \\
\hline 23 & 3 & 5.56 & 38.99 & 29 & 4.77 & 59.50 & 13 & -34.47 \\
\hline 23 & 4 & 7.86 & 1423.60 & 242 & 7.31 & 727.09 & 171 & 48.93 \\
\hline 22 & 4 & 8.59 & 1481.85 & 277 & 7.32 & 396.63 & 107 & 73.23 \\
\hline 22 & 5 & 11.09 & 5433.32 & 1088 & 10.14 & 1546.41 & 476 & 71.54 \\
\hline 21 & 5 & 11.15 & 3569.69 & 815 & 9.29 & 1081.23 & 309 & 69.71 \\
\hline 21 & 6 & 14.16 & 6899.62 & 1524 & 12.64 & 6279.46 & 1402 & 8.99 \\
\hline 20 & 6 & 15.42 & 4242.11 & 1007 & 11.15 & 1000.95 & 296 & 76.40 \\
\hline 20 & 7 & 16.72 & 5144.41 & 1355 & 13.92 & 1514.99 & 614 & 70.55 \\
\hline 19 & 7 & 17.90 & 3581.83 & 1121 & 10.50 & 663.67 & 587 & 81.47 \\
\hline 19 & 8 & 19.31 & 2928.09 & 2604 & 12.17 & 1676.45 & 1542 & 42.75 \\
\hline
\end{tabular}


there are five instances where the improvement in the CPU times with the valid inequalities are over $70 \%$ with a maximum value of $81.47 \%$. The highest improvement in the CPU times using the valid inequalities corresponds to about $1 \mathrm{~h}$.

Lastly, when we compare the number of nodes in the branch and bound tree reported by CPLEX, we observe that fewer nodes and thus less branching are required for the model with the valid inequalities.

In the light of the above observations, since the model is solved more efficiently with the valid inequalities, we include all sets of valid inequalities for the rest of the computational analysis.

Next, we varied the time limit, $T$, between 24 and $18 \mathrm{~h}$, and the total number of hubs to be located, $p$, starting from the first feasible value up to 8 . The results are summarized in Table 2.

The first two columns of Table 2 list the input parameters: $T$ and $p$. The third column presents the optimal objective function value, the fourth and fifth columns present the optimal locations of the ground and airport hubs, respectively. The last column lists the CPU time requirement by CPLEX to solve the corresponding instances to optimality.

Observe from Table 2 that $24 \mathrm{~h}$ service to all of the cities in the Turkish network is possible with two airport hubs located at Ankara (6) and Diyarbakir (21). When we decrease the time limit, more hubs are required to provide the service. For example, one ground hub and four airport hubs are required if each city pair is to receive service within $18 \mathrm{~h}$. Observe also that when $T$ is decreased from 24 to 21 while locating two hubs, the optimal solution results in relocating one hub a short distance (from 21 to 23 ) increasing the cost by less than 1\%. It is not possible to meet the service guarantee of $21 \mathrm{~h}$ without this relocation. With eight hubs, $T$ can be decreased from 24 to 22 at an additional cost of $0.35 \%$ without changing the optimal locations of airport hubs and by relocating one ground hub a short distance (from 5 to 55). Again, it is not possible to give service in $22 \mathrm{~h}$ with eight hubs without this relocation.

Table 2

Results with varying service levels and total number of hubs.

\begin{tabular}{|c|c|c|c|c|c|}
\hline$T(\mathrm{~h})$ & $p$ & Optimal objective function & Locations of ground hubs & Locations of airport hubs & CPU time $(\mathrm{s})$ \\
\hline 24 & 2 & 81489.92 & - & 6,21 & 7.37 \\
\hline 24 & 3 & 73585.66 & - & $6,16,21$ & 101.33 \\
\hline 24 & 4 & 70284.67 & 3 & $6,21,34$ & 582.27 \\
\hline 24 & 5 & 67268.16 & 3,25 & $6,21,34$ & 1167.42 \\
\hline 24 & 6 & 65098.83 & $3,25,27$ & $6,21,34$ & 2627.81 \\
\hline 24 & 7 & 64115.48 & $3,5,25,27$ & $6,21,34$ & 4404.05 \\
\hline 24 & 8 & 63447.84 & $3,5,25,27,68$ & $6,21,34$ & 5996.15 \\
\hline 23 & 2 & 81675.40 & - & 6,21 & 7.98 \\
\hline 23 & 3 & 73771.14 & - & $6,16,21$ & 59.50 \\
\hline 23 & 4 & 70470.15 & 3 & $6,21,34$ & 727.09 \\
\hline 23 & 5 & 67314.99 & 3,25 & $6,21,34$ & 1343.19 \\
\hline 23 & 6 & 65145.67 & $3,25,27$ & $6,21,34$ & 1674.19 \\
\hline 23 & 7 & 64115.48 & $3,5,25,27$ & $6,21,34$ & 5194.47 \\
\hline 23 & 8 & 63447.84 & $3,5,25,27,68$ & $6,21,34$ & 5820.55 \\
\hline 22 & 2 & 82241.50 & - & 6,23 & 3.42 \\
\hline 22 & 3 & 74337.24 & - & $6,16,23$ & 13.10 \\
\hline 22 & 4 & 71036.24 & 3 & $6,23,34$ & 396.63 \\
\hline 22 & 5 & 68503.35 & 3,55 & $6,21,34$ & 1546.41 \\
\hline 22 & 6 & 66334.03 & $3,27,55$ & $6,21,34$ & 2539.84 \\
\hline 22 & 7 & 64340.35 & $3,25,27,55$ & $6,21,34$ & 1969.34 \\
\hline 22 & 8 & 63672.71 & $3,25,27,55,68$ & $6,21,34$ & 4681.59 \\
\hline 21 & 2 & 82241.50 & - & 6,23 & 3.22 \\
\hline 21 & 3 & 74337.24 & - & $6,16,23$ & 10.13 \\
\hline 21 & 4 & 71036.24 & 3 & $6,23,34$ & 281.93 \\
\hline 21 & 5 & 68549.16 & 3 & $6,25,27,34$ & 990.30 \\
\hline 21 & 6 & 67389.10 & $1,3,21$ & $6,25,34$ & 6023.48 \\
\hline 21 & 7 & 66325.32 & $3,21,25,27$ & $6,23,34$ & 6264.64 \\
\hline 21 & 8 & 65176.26 & $3,5,25,27,61$ & $6,21,34$ & 5364.33 \\
\hline 20 & 3 & 81764.88 & - & $6,21,61$ & 7.04 \\
\hline 20 & 4 & 73860.62 & - & $6,16,21,61$ & 56.74 \\
\hline 20 & 5 & 70559.63 & 3 & $6,21,34,61$ & 502.35 \\
\hline 20 & 6 & 68389.50 & 3,65 & $6,25,27,34$ & 983.68 \\
\hline 20 & 7 & 67288.10 & $1,3,21,65$ & $6,25,34$ & 1493.43 \\
\hline 20 & 8 & 66704.72 & $1,3,21,61,65$ & $6,25,34$ & 3714.22 \\
\hline 19 & 4 & 74397.29 & - & $6,16,21,25$ & 6.46 \\
\hline 19 & 5 & 71096.30 & 3 & $6,21,25,34$ & 143.02 \\
\hline 19 & 6 & 69173.53 & 3,27 & $6,21,25,34$ & 316.06 \\
\hline 19 & 7 & 68259.33 & $3,27,68$ & $6,21,25,34$ & 791.71 \\
\hline 19 & 8 & 67675.95 & $3,27,61,68$ & $6,21,25,34$ & 1763.26 \\
\hline 18 & 5 & 72474.53 & 27 & $6,16,21,25$ & 9.29 \\
\hline 18 & 6 & 69173.53 & 3,27 & $6,21,25,34$ & 120.28 \\
\hline 18 & 7 & 68259.33 & $3,27,68$ & $6,21,25,34$ & 398.26 \\
\hline \multirow[t]{2}{*}{18} & 8 & 67675.95 & $3,27,61,68$ & $6,21,25,34$ & 1485.33 \\
\hline & & & & Average & 1704.42 \\
\hline
\end{tabular}


For each service level, when we increase the total number of hubs to be established, the number and the locations of the airport hubs tend to stay the same since the fixed costs of operating airline connections is relatively higher than those of operating truck connections. For each time limit, the fifth column of Table 2 is the same, except at few instances where there is a change in the location of a single airport hub.

When we increase the total number of hubs to be located by one, in almost all of the instances, a new ground hub is established. Compared with the airport hubs, the locations of the ground hubs are more sensitive to the total number of hubs to be located. Clearly, the reluctance to open additional airport hubs is a result of the large fixed costs for airline connections. However, there are exceptions to this. For instance, when $T=21$ and $p=4$, three airport hubs and one ground hub are opened. The minimum cost with $p=5$ is equal to 69223.45 , if we fix the number of airport hubs to three. However, with four airport hubs and one ground hub, the minimum cost is 68549.16 . Hence, when $p$ is increased from four to five, one more airport hub is opened.

When we look at the CPU time requirements, we observe from the last column of Table 2 that the instances with larger $p$ values are relatively harder. In the average, the model is solved in around $28 \mathrm{~min}$ to optimality. The lowest CPU time requirement is about $3.2 \mathrm{~s}$, whereas the highest is less than $1.7 \mathrm{~h}$. In general, the CPU times requirements for the model are reasonable for a network design problem.

Table 2 also presents the optimal objective function value of the corresponding instances. In all of the instances, total costs decrease when the total number of hubs to be located increases. Since the total number of airport hubs usually stays constant, this can be explained with the decrease in the total routing costs with additional ground hubs. The objective function values demonstrate the trade-off between the investment cost of building hubs and the operational costs. With more investment on the total number of hubs to be established, operational costs decrease.

Fig. 3 demonstrates some solutions from Table 2 on the Turkish network (the notation is the same as in Fig. 1). In Fig. 3a-f, only the hub network is depicted, that is, the allocations of the demand nodes to the hub nodes are not shown.

In all of the solutions presented in Fig. 3, there is an airport hub located in Istanbul (34), which is the node generating the highest amount of flow in Turkey. Moreover, again in all of the solutions, at least one airport hub is located in the eastern part of the region in order to provide service within the given time limit.

A comparison of the Fig. $3 \mathrm{~b}$ and $\mathrm{c}$ shows that $\mathrm{a} 1 \mathrm{~h}$ tightening of the delivery time guarantee causes one ground hub to be relocated (a relatively long distance) and to be assigned to a different airport hub. Additionally, a comparison of the Fig. $3 \mathrm{~d}$ and e shows that a $1 \mathrm{~h}$ tightening of the service level causes one airport hub and two ground hubs to be relocated. Similarly, a

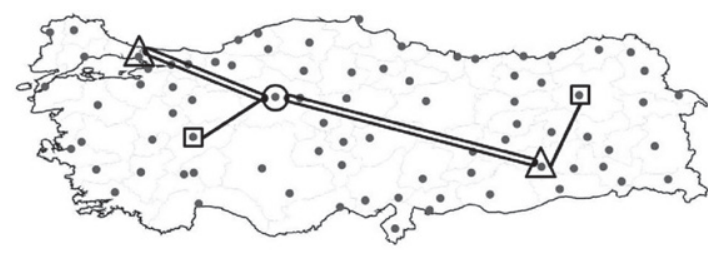

(a) $T=24, p=5$

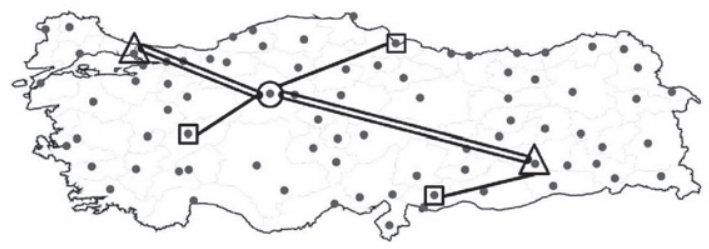

(c) $T=22, p=6$

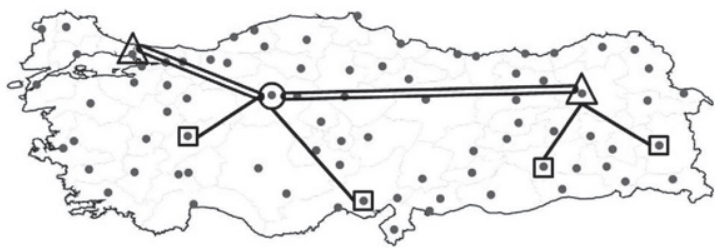

(e) $T=20, p=7$

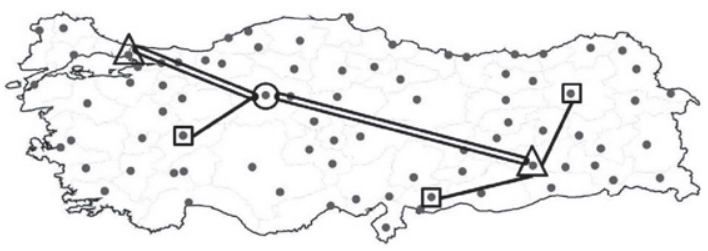

(b) $T=24(23), p=6$

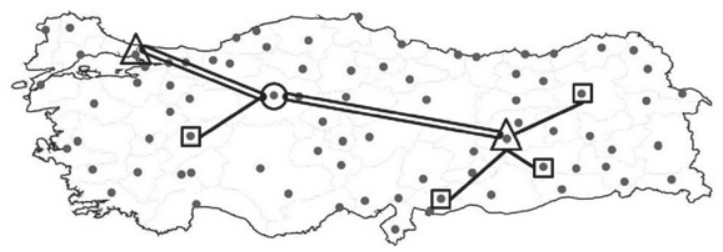

(d) $T=21, p=7$

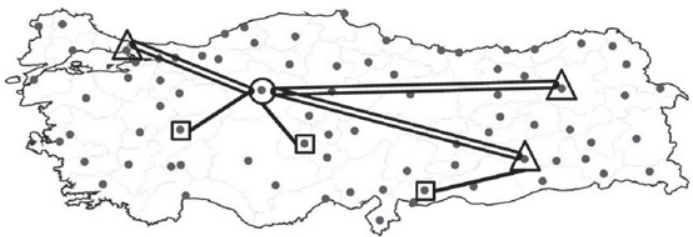

(f) $T=19(18), p=7$

Fig. 3. Results on the Turkish network. 
comparison of the Fig. 3e and f shows that a further one (or two) hour tightening of the delivery time limit causes one ground hub to become an airport hub, and two ground hubs to be relocated.

Observe that the resulting hub networks are tree shaped in Fig. 3a-f. Due to this tree structure, there is a unique path connecting each of the hub nodes. That is, the demand of all the nodes allocated to each of the hub nodes are routed using the unique path between the hub nodes that they are allocated to.

When we analyze the flows on the hub network connections, we observe that most of the demand is transported using the airline connections. This is because unit routing costs on airline connections are negligible compared to fixed costs of operating these connections. Thus, once an airline connection is established the model tries to route as much flow as possible on this airline connection. This result coincides with the actual operational decisions of the cargo company. The company owns its own aircraft to operate on the airline connections and wants to utilize their aircrafts' full capacity.

Even though ground transportation is fast enough to provide service between certain origin-destination pairs within the given time limit, the resulting hub network may force these pairs to employ air transportation. For example, the demand between Ankara and Istanbul can be transported using ground transportation even with the tightest service time bounds. However, in order to give timely service to all the demand nodes allocated to these hubs, and also to achieve economies of scale, the demand between Ankara and Istanbul is routed using the direct airline connection in-between. Nevertheless, note that there are no capacity constraints on the amount of flow carried on the airline connections. This can be considered as a limitation of the current model and should be considered in future research.

As we noted, all the solutions in Fig. 3 resulted in tree shaped hub networks. This is due to fixed costs of operating truck connections. If the fixed costs are lower, more truck connections can be established. Next, we analyze the effects of the fixed costs of operating airline and truck connections on the optimal solutions.

We vary the fixed cost of operating an airline segment between 500 and 10,000. We test two instances with varying airplane operating costs: one where service time limit is set to $24 \mathrm{~h}$ and the number of hubs to be located is six; the other where service time limit is set to $20 \mathrm{~h}$ and the number of hubs to be located is eight. Table 3 summarizes the results.

When the airplane operating costs are at their lowest values, more airport hubs are located and thus, more airline connections are established. This is expected since transportation cost per unit of flow is negligible on the airline connections. When the airline cost increases, the optimal number of airport hubs decreases. Even though we increase airline costs to 10,000 , two airport hubs are still required to provide the service because of the time limit.

We also analyze the effects of the fixed costs of operating truck connections on resulting hub networks. We defined a truck cost coefficient and multiplied the truck operating costs on the allocation connections and on the connections between the hub nodes with this coefficient. We varied the value of this truck cost coefficient from 0 to 10 . Similar to our analysis with the airplane operating costs, we test two instances with varying truck costs. The results are provided in Table 4.

In the sixth column of Table 4, we report the optimal number of truck connections in the resulting hub networks. Observe from Table 4 that with lower values of truck operating costs more truck connections are established. When the fixed costs of operating truck connections are negligible, there is a direct truck connection between all hubs that are allocated to the same airport hub. That is, the sub-graph induced by an airport hub and the ground hubs allocated to it is complete. When we increase the costs of operating truck connections, fewer ground hubs are established in order to have fewer truck connections. With higher truck operating costs the second layer network, connecting airport hubs and the ground hubs allocated to them, is inclined to be a tree.

Note from Table 4 that no matter what the values of the truck operating costs are, a certain number of airport hubs are required at all times in order to provide service within the given service time limit.

Table 3

Results with different values of airline operating costs.

\begin{tabular}{|c|c|c|c|c|c|}
\hline$T(\mathrm{~h})$ & $p$ & Airline cost & Locations of ground hubs & Locations of airport hubs & CPU time $(\mathrm{s})$ \\
\hline 24 & 6 & 500 & - & $1,6,20,21,34,61$ & 6.28 \\
\hline 24 & 6 & 1500 & - & $1,6,20,21,34,61$ & 66.82 \\
\hline 24 & 6 & 2500 & 25,27 & $6,20,21,34$ & 1208.21 \\
\hline 24 & 6 & 3500 & $3,25,27$ & $6,21,34$ & 2627.81 \\
\hline 24 & 6 & 4500 & $3,25,27,34$ & 6,21 & 6409.75 \\
\hline 24 & 6 & 5500 & $3,25,27,34$ & 6,21 & 4819.77 \\
\hline 24 & 6 & 6500 & $3,25,27,34$ & 6,21 & 1791.42 \\
\hline 24 & 6 & 7500 & $3,25,27,34$ & 6,21 & 1114.50 \\
\hline 24 & 6 & 10,000 & $3,25,27,34$ & 6,21 & 590.61 \\
\hline 20 & 8 & 500 & - & $1,6,21,25,34,35,42,55$ & 5.74 \\
\hline 20 & 8 & 1500 & 20,25 & $1,6,21,34,35,55$ & 466.54 \\
\hline 20 & 8 & 2500 & $20,21,65$ & $6,25,27,34,35$ & 2652.76 \\
\hline 20 & 8 & 3500 & $1,3,21,61,65$ & $6,25,34$ & 3714.22 \\
\hline 20 & 8 & 4500 & $1,3,16,21,34,65$ & 6,25 & 4958.18 \\
\hline 20 & 8 & 5500 & $1,3,16,21,34,65$ & 6,25 & 3226.10 \\
\hline 20 & 8 & 6500 & $1,3,16,21,34,65$ & 6,25 & 3010.43 \\
\hline 20 & 8 & 7500 & $1,3,16,21,34,65$ & 6,25 & 1796.82 \\
\hline 20 & 8 & 10,000 & $1,3,16,21,34,65$ & 6,25 & 1190.65 \\
\hline
\end{tabular}


Table 4

Results with different costs for operating truck connections.

\begin{tabular}{|c|c|c|c|c|c|c|}
\hline$T(\mathrm{~h})$ & $p$ & Truck cost coefficient & Locations of ground hubs & Locations of airport hubs & Number of truck connections & CPU time $(\mathrm{s})$ \\
\hline 24 & 6 & 0 & $3,16,34,35$ & 6,21 & 20 & 7155.60 \\
\hline 24 & 6 & 0.25 & $3,25,27$ & $6,21,34$ & 6 & 5442.59 \\
\hline 24 & 6 & 0.5 & $3,25,27$ & $6,21,34$ & 6 & 6232.79 \\
\hline 24 & 6 & 1 & $3,25,27$ & $6,21,34$ & 6 & 2627.81 \\
\hline 24 & 6 & 5 & 25 & $1,6,20,21,34$ & 2 & 51.73 \\
\hline 24 & 6 & 10 & - & $1,6,20,21,34,61$ & 0 & 7.08 \\
\hline 20 & 8 & 0 & $1,3,16,34,35,65$ & 6,25 & 32 & 4461.35 \\
\hline 20 & 8 & 0.25 & $1,3,21,42,65$ & $6,25,34$ & 14 & 7061.89 \\
\hline 20 & 8 & 0.5 & $1,3,16,21,65$ & $6,25,34$ & 10 & 6059.49 \\
\hline 20 & 8 & 1 & $1,3,21,61,65$ & $6,25,34$ & 10 & 3714.22 \\
\hline 20 & 8 & 5 & $25,27,68$ & $6,20,21,34,55$ & 6 & 105.97 \\
\hline 20 & 8 & 10 & 68 & $6,20,21,25,27,34,55$ & 2 & 11.67 \\
\hline
\end{tabular}

Table 5

Results with different central airport hub locations.

\begin{tabular}{|c|c|c|c|c|c|c|c|c|c|c|}
\hline \multirow{2}{*}{$\begin{array}{l}T \\
(\mathrm{~h})\end{array}$} & \multirow[t]{2}{*}{$p$} & \multicolumn{3}{|c|}{ Ankara (06) } & \multicolumn{3}{|c|}{ Istanbul (34) } & \multicolumn{3}{|c|}{ Kayseri (38) } \\
\hline & & $\begin{array}{l}\text { Objective } \\
\text { function }\end{array}$ & $\begin{array}{l}\text { Locations of } \\
\text { ground hubs }\end{array}$ & $\begin{array}{l}\text { Locations of } \\
\text { airport hubs }\end{array}$ & $\begin{array}{l}\text { Objective } \\
\text { function }\end{array}$ & $\begin{array}{l}\text { Locations of } \\
\text { ground hubs }\end{array}$ & $\begin{array}{l}\text { Locations of } \\
\text { airport hubs }\end{array}$ & $\begin{array}{l}\text { Objective } \\
\text { function }\end{array}$ & $\begin{array}{l}\text { Locations of } \\
\text { ground hubs }\end{array}$ & $\begin{array}{l}\text { Locations of } \\
\text { airport hubs }\end{array}$ \\
\hline 24 & 2 & 81489.92 & - & 6,21 & 85223.43 & - & 23,34 & Infeasible & - & - \\
\hline 24 & 3 & 73585.66 & - & $6,16,21$ & 74610.45 & - & $6,23,34$ & 73460.62 & - & $16,21,38$ \\
\hline 24 & 4 & 70284.67 & 3 & $6,21,34$ & 70695.81 & - & $20,21,34,38$ & 70695.81 & - & $20,21,34,38$ \\
\hline 24 & 5 & 67268.16 & 3,25 & $6,21,34$ & 67314.99 & 3,25 & $6,21,34$ & 67983.39 & 25 & $20,21,34,38$ \\
\hline 24 & 6 & 65098.83 & $3,25,27$ & $6,21,34$ & 65145.67 & $3,25,27$ & $6,21,34$ & 66846.25 & 6,25 & $20,21,34,38$ \\
\hline 24 & 7 & 64115.48 & $3,5,25,27$ & $6,21,34$ & 64115.48 & $3,5,25,27$ & $6,21,34$ & 65881.12 & $1,6,25$ & $20,21,34,38$ \\
\hline 24 & 8 & 63447.84 & $3,5,25,27,68$ & $6,21,34$ & 63447.84 & $3,5,25,27,68$ & $6,21,34$ & 65139.99 & $1,6,25,34,35$ & $16,21,38$ \\
\hline 20 & 3 & 81764.88 & - & $6,21,61$ & Infeasible & - & - & 73460.62 & - & $16,21,38$ \\
\hline 20 & 4 & 73860.62 & - & $6,16,21,61$ & 74670.50 & - & $6,21,25,34$ & 70695.81 & - & $20,21,34,38$ \\
\hline 20 & 5 & 70559.63 & 3 & $6,21,34,61$ & 71096.30 & 3 & $6,21,25,34$ & 68336.81 & 25 & $20,21,34,38$ \\
\hline 20 & 6 & 68389.50 & 3,65 & $6,25,27,34$ & 69173.53 & 3,27 & $6,21,25,34$ & 67199.67 & 6,25 & $20,21,34,38$ \\
\hline 20 & 7 & 67288.10 & $1,3,21,65$ & $6,25,34$ & 68259.33 & $3,27,68$ & $6,21,25,34$ & 66234.54 & $1,6,25$ & $20,21,34,38$ \\
\hline 20 & 8 & 66704.72 & $1,3,21,61,65$ & $6,25,34$ & 67675.95 & $3,27,61,68$ & $6,21,25,34$ & 65493.41 & $1,6,25,34,35$ & $16,21,38$ \\
\hline
\end{tabular}

Observe from the CPU time requirements that, in general, the problems with higher values of truck operating costs require less time to be solved to optimality.

Lastly, we want to analyze the influence of the location of the central airport hub on the resulting solutions. Remember that in all of our previous experiments, the central airport hub is located in Ankara where the cargo company has its major sorting center. For this analysis, we select two locations for the central airport hub other than Ankara. The first location we select is Istanbul (34), which is the city generating the highest amount of demand in Turkey. The other location we test is Kayseri (38) which is located geographically close to the center of the Anatolian region. We test two service time limits with different central airport hub locations. The results are summarized in Table 5.

For each time limit and $p$ value, Table 5 presents the optimal objective function values and the optimal locations of ground and airport hubs corresponding to different central airport hub locations. For convenience, we present our previous results, when the central airport hub is located in Ankara, in columns 3-5 in Table 5.

Observe from the optimal objective function values with $T=24$ that, except when $p=3$, the lowest objective function values in Table 5 are attained when the central airport hub is located in Ankara. When $T=20$, on the other hand, the lowest objective function values correspond to the instances when the central airport hub is located in Kayseri. Note that for $T=20$ and $p=2$, the objective function value of Kayseri is $11.3 \%$ better than Ankara. This shows that sometimes cost difference can be significant.

Note from Table 5 that some $p$ values are infeasible when the location of the central airport hub is altered. This is due to service time limit. For example, when the central airport hub is located in Kayseri, the time limit cannot be satisfied with only two hubs. Because of the location of Kayseri, at least two air hub links are required to give timely service. Similarly, when time limit is set to $20 \mathrm{~h}$, it is not possible to provide service with three hubs when the central airport hub is located in Istanbul. Observe from Table 5 that at least three air links are required to provide service in $20 \mathrm{~h}$ when the central airport hub is located in Istanbul.

In our example, Ankara was already selected by the company as the central airport hub due to land availability and some other managerial issues. The results in Table 5 reveals that an analysis on the location of the central airport hub could also be advantageous in terms of transportation and operational costs. 


\section{Conclusions}

In this paper, we introduced a new hub location problem motivated by the network structure of a cargo delivery company that operates a multimodal and hierarchical hub network. The aim of our problem is to design a minimum cost two-level hub network such that each pair of demand nodes receives service within a predetermined time bound.

A linear mixed integer programming model is derived and some variable fixing rules and valid inequalities are proposed. Comprehensive computational experiments are presented on the Turkish network data set with the proposed hierarchical multimodal hub location model with time-definite deliveries. The model is solved efficiently to optimality using the commercial solver CPLEX.

The contributions of this paper to the literature are firstly to show that with different service levels, the locations of the airport hubs tend to stay the same even though the total number of hubs to be located is increased. Compared with the airport hubs, the locations of the ground hubs are more sensitive to the total number of hubs to be located. Secondly, to show that it is possible to obtain better service levels at little additional costs.

Another contribution of this paper to the literature is to demonstrate the trade-off between investment and operational costs and to show that with more investment on the total number of hubs to be established, total transportation and operational costs decrease. The decision maker should consider this trade-off while designing the hub network.

We show that when the costs of operating truck connections are increased, fewer ground hubs are established in order to have fewer truck connections. In addition, the optimal hub network is inclined to be a tree. If the fixed costs of operating truck connections are lower, on the other hand, more truck links are established.

We test and compare different locations for the central airport hub. Our results show that different central airport hub locations can be advantageous under different service time promises. Hence, the location of the central airport hub can also be determined optimally once the service level is set.

\section{References}

Alumur, S., Kara, B.Y., 2008. Network hub location problems: the state of the art. European Journal of Operational Research 190, 1-21.

Alumur, S., Kara, B.Y., 2009. A hub covering network design problem for cargo applications in Turkey. Journal of the Operational Research Society 60, 13491359.

Alumur, S.A., Kara, B.Y., Karasan, O.E., 2009. The design of single allocation incomplete hub networks. Transportation Research Part B 43, 936-951.

Alumur, S.A., Kara, B.Y., Karasan, O.E., 2012. Multimodal hub location and hub network design. OMEGA 40, 927-939.

Arnold, P., Peeters, D., Thomas, I., 2004. Modelling a rail/road intermodal transportation system. Transportation Research Part E 40, 255-970.

Beasley, J.E., 1990. OR-Library: Hub location <http://people.brunel.ac.uk/mastjjb/jeb/orlib/phubinfo.html> (accessed 15.03.2010).

Boland, N., Krishnamoorthy, M., Ernst, A.T., Ebery, J., 2004. Preprocessing and cutting for multiple allocation hub location problems. European Journal of Operational Research 155, 638-653.

Calik, H., Alumur, S.A., Kara, B.Y., Karasan, O.E., 2009. A tabu-search based heuristic for the hub covering problem over incomplete hub networks. Computers and Operations Research 36, 3088-3096.

Campbell, J.F., 1994. Integer programming formulations of discrete hub location problems. European Journal of Operational Research 72, 387-405.

Campbell, J.F., 1996. Hub location and the p-hub median problem. Operations Research 44, 1-13.

Campbell, J.F., 2009. Hub location for time definite transportation. Computers and Operations Research 36, 3107-3116.

Campbell, J.F., Ernst, A.T., Krishnamoorthy, M., 2002. Hub location problems. In: Drezner, Z., Hamacher, H. (Eds.), Facility Location: Applications and Theory. Springer-Verlag, Berlin.

Campbell, J.F., Ernst, A.T., Krishnamoorthy, M., 2005a. Hub arc location problems: part I - introduction and results. Management Science 51, $1540-1555$.

Campbell, J.F., Ernst, A.T., Krishnamoorthy, M., 2005b. Hub arc location problems: part II - formulations and optimal algorithms. Management Science 51, $1556-1571$.

Chen, H., Campbell, A.M., Thomas, B.W., 2008. Network design for time-constrained delivery. Naval Research Logistics 55, 493-515.

Contreras, I., Fernández, E., Marín, A., 2009. Tight bounds from a path based formulation for the tree of hub location problem. Computers and Operations Research 36, 3117-3127.

Contreras, I., Fernández, E., Marín, A., 2010. The tree of hubs location problem. European Journal of Operational Research 202, $390-400$.

Ernst, A.T., Krishnamoorthy, M., 1996. Efficient algorithms for the uncapacitated single allocation p-hub median problem. Location Science 4, 139-154.

Ernst, A.T., Krishnamoorthy, M., 1998. Exact and heuristic algorithms for the uncapacitated multiple allocation p-hub median problem. European Journal of Operational Research 104, 100-112.

Ernst, A.T., Jiang, H., Krishnamoorthy, M., 2005. Reformulations and computational results for uncapacitated single and multiple allocation hub covering problems. Unpublished Report, CSIRO Mathematical and Information Sciences, Australia.

Ernst, A.T., Hamacher, H., Jiang, H., Krishnamoorthy, M., Woeginger, G., 2009. Uncapacitated single and multi allocation p-hub center problems. Computers and Operations Research 36, 2230-2241.

Gourdin, E., Labbé, M., Yaman, H., 2002. Telecommunication and location. In: Drezner, Z., Hamacher, H.W. (Eds.), Facility Location: Applications and Theory. Springer, pp. 275-305.

Hamacher, H.W., Labbé, M., Nickel, S., Sonneborn, T., 2004. Adapting polyhedral properties from facility to hub location problems. Discrete Applied Mathematics 145, 104-116.

Ishfaq, R., Sox, C.R., 2011. Hub location-allocation in intermodal logistic networks. European Journal of Operational Research 210, 213-230.

Kara, B.Y., Tansel, B.C., 2000. On the single-assignment p-hub center problem. European Journal of Operational Research 125, 648-655.

Kara, B.Y., Tansel, B.C., 2001. The latest arrival hub location problem. Management Science 47, 1408-1420.

Kara, B.Y., Tansel, B.C., 2003. The single-assignment hub covering problem: models and linearizations. Journal of the Operational Research Society 54, 5964.

Klincewicz, J.G., 1998. Hub location in backbone/tributary network design: a review. Location Science 6, 307-335.

Labbé, M., Yaman, H., 2004. Projecting the flow variables for hub location problems. Networks 44, 84-93.

Labbé, M., Yaman, H., 2008. Solving the hub location problem in a star-star network. Networks 51, 19-33.

Labbé, M., Yaman, H., Gourdin, E., 2005. A branch and cut algorithm for hub location problems with single assignment. Mathematical Programming 102, 371-405.

Limbourg, S., Jourquin, B., 2009. Optimal rail-road container terminal locations on the European network. Transportation Research Part E 45, 551-563.

Lin, C.-C., 2010. The integrated secondary route network design model in the hierarchical hub-and-spoke network for dual express services. International Journal of Production Economics 123, 20-30. 
Lin, C.-C., Chen, S.-H., 2004. The hierarchical network design problem for time-definite express common carrier. Transportation Research B 38, $271-283$. Marín, A., 2005. Formulating and solving splittable capacitated multiple allocation hub location problems. Computers and Operations Research $32,3093-$ 3109.

Marín, A., Canovas, L., Landete, M., 2006. New formulations for the uncapacitated multiple allocation hub location problem. European Journal of Operational Research 172, 274-292.

Meng, Q., Wang, X., 2011. Intermodal hub-and-spoke network design: incorporating multiple stakeholders and multi-type containers. Transportation Research Part B 45, 724-742.

Meyer, T., Ernst, A.T., Krishnamoorthy, M., 2009. A 2-phase algorithm for solving the single allocation p-hub center problem. Computers and Operations Research 36, 3143-3151.

Nickel, S., Schobel, A., Sonneborn, T., 2001. Chapter 1: hub location problems in urban traffic networks. In: Niittymaki, J., Pursula, M. (Eds.), Mathematics Methods and Optimization in Transportation Systems. Kluwer Academic Publishers, pp. 1-12.

O'Kelly, M.E., 1986. The location of interacting hub facilities. Transportation Science 20, 92-105.

O’Kelly, M.E., 1987. A quadratic integer program for the location of interacting hub facilities. European Journal of Operational Research 32, 393-404.

O'Kelly, M.E., Lao, Y., 1991. Mode choice in a hub-and-spoke network: a zero-one linear programming approach. Geographical Analysis 23, 283-397.

O’Kelly, M.E., Bryan, D., Skorin-Kapov, D., Skorin-Kapov, J., 1996. Hub network design with single and multiple allocation: a computational study. Location Science 4, 125-138.

Racunica, I., Wynter, L., 2005. Optimal location of intermodal freight hubs. Transportation Research Part B $39,453-477$.

Şahin, G., Süral, H., 2007. A review of hierarchical location models. Computers \& Operations Research 34, $2310-2331$.

Sim, T., Lowe, T.J., Thomas, B.W., 2009. The stochastic p-hub center problem with service-level constraints. Computers \& Operations Research 36, 31663177.

Smilowitz, K.R., Daganzo, C.F., 2007. Continuum approximation techniques for the design of integrated package distribution systems. Networks 50, 183196.

Sohn, J., Park, S., 1998. Efficient Solution procedure and reduced size formulations for p-hub location problems. European Journal of Operational Research 108, 118-126.

Tan, P.Z., Kara, B.Y., 2007. A hub covering model for cargo delivery systems. Networks 49, 28-39.

Wagner, B., 2008. Model formulations for hub covering problems. Journal of the Operational Research Society 59, 932-938.

Yaman, H., 2009. The hierarchical hub median problem with single assignment. Transportation Research Part B 43, 643-658.

Yaman, H., 2011. Allocation strategies in hub networks. European Journal of Operational Research 211, 442-451.

Yaman, H., Kara, B.Y., Tansel, B.C., 2007. The latest arrival hub location problem for cargo delivery systems with stopovers. Transportation Research Part B 41, 906-919.

Yaman, H., Karasan, O.E., Kara, B.Y., 2012. Release time scheduling and hub location for next day delivery. Operations Research.

Yoon, M.G., Current, J., 2008. The hub location and network design problem with fixed and variable arc costs: formulation and dual-based solution heuristic. Journal of the Operational Research Society 59, 80-89. 